10 years ESJ

Special edition

\title{
Context, Cognition, and Communication: Understanding how the Psychology of Location Affects Health and Medical Communication
}

\author{
Kirk St.Amant, PhD \\ Louisiana Tech University and University of Limerick, U.S.A.
}

\section{Doi: $10.19044 /$ esj.2021.v17n30p8}

Submitted: 25 June 2021

Accepted: 12 July 2021

Published: 10 September 2021
Copyright 2021 Author(s)

Under Creative Commons BY-NC-ND

4.0 OPEN ACCESS

Cite As:

St.Amant K. (2021). Context, Cognition, and Communication: Understanding how the Psychology of Location Affects Health and Medical Communication. European Scientific Journal, ESJ, 17 (30), 8. https://doi.org/10.19044/esj.2021.v17n30p8

\section{Abstract}

Prior research reveals that cognition - or how the mind processes information - often guides human behavior in familiar settings (St.Amant, 2018). Such factors can affect the communication practices persons use to convey information about health concepts or medical processes (Hamm, 2003; St.Amant, 2021). The challenge becomes anticipating such connections in order to create texts and visuals audiences can understand and use to meet healthcare needs. This article presents an approach to meeting this need by applying the concept of cognitive scripts to understand and address the communication expectations audiences often associated with health and medical settings. In presenting these ideas, the article begins with an explanation of how cognitive scripts can affect communication processes. From there, the author advocates applying script dynamics to health and medical communication practices. To do so, the author expands upon ideas in the literature on cognitive scripts to create a script-based approach for researching an audience's expectations of healthcare situations. The author then describes how to apply the information collected from this research to create communication materials audiences can more easily use in healthcare contexts. The result is a three-factor method that focuses on applying cognitive scripts to identify and address an audience's expectations for healthcare communication in a given context. Readers can then use this approach to design healthcare communication materials that audiences can easily and effectively use. 
Keywords: Cognition, Communication, Design, Expectations, Script

\section{Introduction}

Successful healthcare generally requires effective communication (Heifferon, 2004). What constitutes effective communication in health and medical contexts, however, is not fixed. Rather, it varies from audience to audience based upon experiences (Ku \& Lupton, 2020; Jones, 2013). Central to these dynamics are the cognitive scripts the mind uses to process information and engage in activities (St.Amant, 2017a). As scripts affect how audiences conceptualize messages, they can greatly affect communication in healthcare situations (St.Amant, 2018). The better individuals understand such scripts, the more effectively they can convey health and medical information to different audiences.

This article examines what cognitive scripts are and how they shape practices for communicating about healthcare. The article also reviews the ways scripts can cause miscommunication and affect how audiences engage in healthcare practices. In researching these topics, the author reviewed the literature on cognitive scripts in order to identify different trends and dynamics in relation to how scripts affect communication practices in different situations and settings. The result of this review is the description of script-related processes discussed in the first part of this entry.

Based upon the how scripts affect cognition and interaction, the author developed an approach for applying script theory - and the elements in scripts - to research the scrips audiences use to conceptualize and communicate about healthcare (discussed in the next part of the article). The author then concludes the article by describing how to use this approach to engage in effective healthcare communication with different audiences. Ideally, readers can apply these ideas to enhance the success with which they share healthcare information across groups in different contexts.

\section{Cognition, Attention, and Action}

The way our minds guide our actions is often connected to the location where we perform an activity (Tomkins, 1978 \& 1987; St.Amant, 2017a). When we enter a setting, for example, we usually scan our surroundings to determine where we are. Once we identify the location, we automatically behave in a certain way - including expecting to encounter and use particular things and to interact with particular individuals in that setting (St.Amant, 2018). So powerful is this connection between place and behavior that we rarely notice the dozens of tasks we reflexively perform in a setting. Conversely, when we cannot identify our location, confusion and panic often result, and many of us feel unable to do anything until we know where we are. 
These dynamics connect to psychological mechanism called a cognitive script (or script), and our brains use these scripts to process information easily and effectively.

Scripts help our brains organize information associated with the locations where we regularly perform certain tasks (Tomkins, 1978; St.Amant, 2017a \& 2018). This situation arises because the human brain is limited in the information it can process at one time (Sweller, 1988; Paas, Renkl, \& Sweller, 2004 ). To address this limitation, our brains have developed mechanisms for combining different pieces of information into overall units, or chunks, for easier processing (Ratey, 2002). So, instead of viewing a location as multiple distinct items to engage with individually, our minds combine these items into a single unit to process quickly and easily (Sweller, 1988; Tse et al., 2007; Yamada \& Itsukushima, 2013). As a result, we do not perceive a location as a setting comprised of many different objects and individual. Rather, we conceptualize it as a single unit of information for our minds to address (St.Amant, 2017a \& b). For example, instead of perceiving a medical examining room as a number of different items or parts, our minds learn to combine those parts into one unit for processing (i.e., an examining room).

In combining information into units, scripts highlight the items and individuals that play an essential role in a setting (Schank \& Abelson, 1977; St.Amant, 2017a \& 2018). Such an approach is important because cognitive limitations make it difficult for our minds to simultaneously keep track of all items in a location. (That is often too much information for our brains to process at one time.) Rather, scripts train our minds to focus only on those things that play a central role in the actions we perform in a location setting. In this way, scripts minimize the items we need to focus on in a location (Sweller, 1988; Paas, Renkl, \& Sweller, 2004; Postle, 2016). This situation explains why we can enter a location knowing exactly what we will find and who we will interact with there. It also explains why we pay attention to certain details of a location, but ignore others (St.Amant, 2018). Many of us, for example, expect to encounter a special examining table in a medical examining room; however, we are often unaware of how many electrical outlets that room might have - or if it will have electrical outlets at all.

While the specific items in a script can vary, most scripts organize information into general categories that guide our activities. These categories encompass

- Why we are in a location (e.g., the objective we wish to achieve in that setting)

- What activities we associate with achieving our objective in that location

- Which items we expect to use to perform these activities in that location 
- Who will perform or participate in certain activities in that location

- How we expect to use - and expect others to use-items to perform tasks in that setting

(Tomkins, 1978 \& 1987; Schank \& Abelson, 1977; Hamm, 2003; Gavin\& Hockey, 2010)

These elements become the factors we either expect to encounter and use or that play a role in the activities we associate with a setting (St.Amant, 2017a \& 2018). Our script for examining room, for example, is likely comprised of "physician," "examining table," "bandages," etc. This script likely also contains expectations of who will perform certain activities and how they will perform them during a medical examination. As a result, when we enter an examining room, we know what items to focus on and use (e.g., sitting on an examining table and letting the physician examine us). We also know what items to ignore in that setting (e.g., electrical outlets, trim on the walls, handles on cabinets, etc.). These dynamics limit what we need to focus our attention on in a location and reduce the items our minds need to process in a setting (Sweller, 1988; Postle, 2007; Yamada \& Itsukushima, 2013).

\section{Experience, Expectations, and Variations}

We are not born with the scripts that guide our activities in a setting. Rather, we develop them based on repeated experiences in a location over time (Sweller, 1988; Tse et al., 2007; Yamada \& Itsukushima, 2013). The process works as follows: The first time we enter a new place (e.g., an examining room in a hospital), we do not know what to focus our attention on, for we do not know what will occur (and how) in that setting. As a result, we generally feel overwhelmed and nervous as our attention moves from item to item trying to determine what to focus on in order to perform tasks in that context.

Over time, we learn which activities will occur in that setting. We also learn what we should do in that location, what objects to use, who to interact with, and what actions those persons will perform (Schank \& Abelson, 1977; St.Amant, 2017a \& 2018). Through these experiences, we learn to associate certain actions, objects, and individuals with that setting. Our minds then combine these factors into a script we use to perform activities in that location (Tomkins, 1978 \& 1987; Schank \& Abelson, 1977; Hamm, 2003; Gavin \& Hockey, 2010). Once formed, these scripts begin to operate on a subconscious level. As a result, once we recognize where we are, we instantly know what to do, to use, to expect, etc. (Ratey, 2002). This sub-conscious processing frees our conscious minds to focus on new activities in a location - activities like having conversations with others. The sub-conscious operation of scripts is why we often seem to behave reflexively in known locations (Ratey, 2002). 
Because scripts are based on experiences, individuals can have different scripts for the same process (St.Amant, 2017a). One such difference involves assumptions about the location where a particular process occurs (St.Amant, 2018). If, for example, you go to the hospital for a physical examination, you expect that process to happen in a certain way (e.g., you sit on an examining table and allow a physician to perform certain diagnostic tasks). You also expect to encounter specific individuals who participate in these activities and use particular items (e.g., a physician who uses a stethoscope to assess your heartbeat). Other audiences, by contrast, might associate the physical examination process with a different location - such as the living room of their homes. This different setting might have different parameters that affect what individuals expect to occur in that location (e.g., sit in a chair in their living room). Such factors might also mean individuals have different expectations of who they will interact in that location and what such interactions entail (e.g., allow a visiting nurse to check one's heartbeat by placing two fingers on the individual's wrist).

A second script variation involves the elements used to perform processes in a setting (St.Amant, 2017a \& 2018). Different audiences might associate the same location with a process - such as associating a physical examination with the examining room of a hospital. These audiences, however, could have different expectations of what actions, items, and persons they consider central to performing activities in that setting (St.Amant, 2017b). One audience's script for physical examination, for example, might expect a physician to check the patient's heart rate with a stethoscope during the physical exam. Yet another audience's experiences might have taught individuals that a nurse will use a digital heart monitor to perform this process before the patient meets with a physician. Thus, even if audiences associate the same location with a healthcare process, the scripts they use to conceptualize that process might differ (e.g., who performs an activity and how in a setting). Such differences in script-based assumptions can have important implications for how we communicate about healthcare.

\section{Communication and Confusion}

Scripts - and script-related variations - often affect how we share information on healthcare topics and medical processes. This is because scripts play a central role in how we use words and images to communicate ideas to others. Whenever we wish to communicate an idea to others, our mind generally accesses the script we associate with the related process. We then use words to describe the dynamics of that script or attempt to re-create those dynamics in visuals (Lindstrom, 2010; Eyal, 2014). It is a process similar to watching a movie and using words to describe what we observe or attempting to re-create these mental representations via visuals. With written 
texts, we assume audiences will use our words to mentally re-create that same depiction and then replicate the actions in that representation. For images, we assume the audience will be in a setting similar to the visual we created and be able to replicate activities as depicted in that image or images.

These factors mean that miscommunication can occur when the script of the writer or artist does not match that of the audience using a text or images (St.Amant, 2017a \& 2018). In some cases, these differences might result in writers or artists depicting processes as taking place in a location that does not align with the audience's experiences (St.Amant, 2017b). Per the physical examination example, confusion could occur if the writer/artist depicts that process as occurring in a hospital, but the audience actually performs that process in their own living rooms. In this instance, the audience might consider the information provided unusable because it does not reflect the location where audience members perform that activity (St.Amant, 2017b).

In other cases, the writer/artist and audience might associate the same location with the script for a healthcare process. The dynamics of performing activities in that location, however, could vary based on differing experiences (St.Amant, 2017a \& 2018). For example, the actions audiences expect the patient vs. a healthcare provider to perform during process can differ (e.g., patients check their own weight before meeting with physician vs. physician checks patient's weight during exam). In other cases, what items individuals expect to use - or allow others to use on them - during a process can differ (e.g., using a digital blood pressure cuff vs. a manual sphygmomanometer to check blood pressure during a physical exam). Such differences could lead to confusion if individuals are asked to do something or to use something unfamiliar. These disconnects could result in a lack of trust as audiences might consider information inadequate because it does not reflect their expectations for performing a process in a location (St.Amant, 2017b). The results could be detrimental if audiences refuse to use information or attempt to revise a process to reflect the audience's expectations.

\section{Comprehension, Content, and Contextualization}

The objective of health and medical communication is to provide audiences with content (i.e., words and images) they will accept and can use to perform a healthcare process (Heifferon, 2004; Jones, 2013; Ku \& Lupton, 2020). Achieving this objective involves understanding the scripts that guide how individuals create, interpret, and use such content. To do so, writers and artists must provide information in a way that addresses the script expectations an audience associates with a healthcare activity (St.Amant, 2017a \& 2018). Thus, the first step in healthcare communication is not to create content, but to identify the script(s) - and related script elements - an audience associate with a healthcare process (Heifferon, 2004; Jones, 2013; Ku \& Lupton, 2020). 
Known as script mapping, the process begins by identifying the core elements of the scripts an audience associates with a healthcare process (St.Amant 2017a \& 2018). Such mapping requires meeting with members of the intended audience in order to identify that audience's script expectations for a process. Collecting such information can involve

- Individual interviews with multiple members of the intended audience to gain individual perspectives on what scripts for a healthcare process entail (Schwab, 2016; InterQ, 2021)

- Focus group meetings where 5-7 members of an audience discuss common script expectations and experiences associated with an activity (Schwab, 2016; InterQ, 2021)

Each method identifies certain information (individual vs. group perspectives), and both approaches can help map the scripts an audience associates with certain healthcare activities.

The objective of both approaches is to collect information on what factors - locations, individuals, items, and activities - an audience associates with the script for a healthcare process (St.Amant, 2017a \& 2018). Mapping script expectations should therefore include both interviews and focus groups when possible. The goal of these activities is to collect information from a multiple individuals in order to identify the common elements audience members associate with certain healthcare processes. (The number of interviews and focus groups will likely depend on the time and funding available for conducting such research.)

Researchers can use the resulting information to identify common audience expectations for the following script elements:

- The location an audience associate with a process

- The persons assumed to perform certain activities during this process

- The actions the audience members assume they will perform during this process

- The items that audience members and that others will use to perform these activities

This mapping helps identify the scripts audiences use to conceptualize healthcare processes. Writers and artists can use this information to present concepts in a way that helps audiences understand ideas according to the scripts they have for a healthcare process. The goal is to help audiences contextualize information - or the content provided - into their existing scripts for a healthcare process. 
To facilitate such contextualization, content creators (i.e., writers and artists) need to review an audience's script expectations and determine three (3) factors before creating healthcare content for an audience:

Factor 1: What content needs to be added in order to help audience members conceptualize ideas and activities

In some cases, an updated or new healthcare process might require individuals to add a new activity to existing activities (e.g., wearing masks in a hospital to prevent the spread of COVID-19) (St.Amant, 2017b). Such an addition requires audience members to modify their existing script for a process in order to conceptualize this new information within the context of a known activity or setting (St.Amant, 2017b \& 2018).

To address this factor, content creators should provide information that explains

- What this overall activity is (e.g., "Wearing Masks to Maintain Safety")

- Where it occurs (e.g., "To be done every time you visit a healthcare facility")

- Who performs this process (e.g., "To be done by all individuals who visit a healthcare facility")

- Why individuals need to perform this activity (e.g., "Wearing a mask reduces the transmission of airborne pathogens")

- How to perform the activity in the related setting (e.g., "To put on a mask when visiting a healthcare facility, perform the following steps:" or "A worker at the facility will help you put on a mask in the following way:")

- Who provides items needed to perform the process in that setting (e.g., "You are expected to obtain a mask in advance of visiting a healthcare facility. You can purchase masks at ..." or "Healthcare facilities will provide individuals with masks at kiosks located in the entrances of the facility.")

- Such information helps audiences contextualize new actions and ideas by explaining how new items fit into the existing script audience members have for a healthcare process in a location.

Factor 2: What content requires revision or removal because it does not reflect the location where an audience perform a process

Certain healthcare activities can require individuals to have a particular expertise, have access to certain items, or both to perform a process correctly (or at all) in a location (St.Amant, 2017b). Scrip mapping, however, might 
reveal that the location where an audience receives certain care does not provide access to

- Materials needed to perform a process (e.g., syringes for drawing blood)

- Individuals with the expertise needed to perform an activity (e.g., drawing blood)

or both. In such situations, achieving a particular healthcare objective might require the use of an alternative process to provide certain care in that context (e.g., using clothing to cover one's nose and mouth if a medical mask is not available). In such cases, content creators would need to research other options for providing a certain kind of care in a particular context. They could then create content that present such alternative approaches to an intended audience. Ideally, this content would allow audience members to achieve a healthcare objective in the setting associated with their script for that process.

Occasionally, research could reveal that certain activities are not viable in a setting (e.g., individuals cannot access technologies for measuring blood pressure) (St.Amant, 2017b). In such instance, content creators might need to omit information on such processes in order to avoid causing confusion among audience members (e.g., omitting information on blood pressure from instructions explaining how to do a medical diagnostic). Alternatively, content creators could provide audiences with an alternative for achieving a healthcare objective at a different time and in another location (e.g., requesting patients visit a clinic to have their blood pressure checked).

Factor 3: What completely new content needs to be created because an audience expects a processes to occur in a different setting.

Script mapping could reveal that an audience expect a healthcare process to occur in a location that is completely different from what the content creator had assumed (St.Amant, 2017a \& 2017b). Such differences could mean new content is needed to address alternate approaches for performing a healthcare process in a location. The resulting content would likely also need to account for different items and individuals available in that setting. Such factors mean that creating effective content requires more than modifying information to address certain differences in relatively common scripts.

In such situations, completely new content is often needed to present approaches that reflect the realities of how an audience can achieve a healthcare objective in a particular location (St.Amant, 2017b). Access to antibiotics, for example, could be restricted in certain settings. As a result, processes based upon scheduling and administering regular, low doses of an antibiotic over time might not be possible in a location. Addressing this factor in order to provide the related care (i.e., administering antibiotics to treat an infection) might require content creators to work with healthcare providers and 
develop a different approach that is safe and that addresses the related healthcare objective (e.g., a process for administering a single large dose of antibiotics to treat an infection). Content creators would then create materials that communicate this approach to audiences seeking the related care in such contexts.

The script-based differences noted here can be deceptively simple. Failing to identify and address them, however, can result in the creation of healthcare content audiences misunderstand, misuse, or fail to use due to differing script expectations. The better content creators understand the script factors influencing a healthcare situation, the more effectively they can contextualize information to provide audiences with healthcare content.

\section{Contextualizing Content for COVID-19 and Beyond}

Contextualizing content according to scripts has been central to addressing healthcare challenges during the global COVID-19 pandemic (St.Amant, 2021). In many cases, the scripts for daily activities needed to change in order to address public health needs. These changes included contextualizing new items into many everyday routines - such as providing instructions on when and how to wear masks in public places (Your Guide to Masks, 2021). They also involved providing audiences with information, such as instructions on social distancing, to help revise existing scripts for interacting with others in familiar settings (Social Distancing, 2020). Many of these activities were neither complicated nor particularly dramatic, yet they differed from the scripts most audiences used to engage in processes across various locations. As a result, effectively communicating about such healthcare practices involved identifying where such activities were missing from existing scripts and providing content to help audiences contextualize these new activities into those scripts (St.Amant, 2021).

In other instances, effective healthcare communication involved creating content based on completely new scripts for novel practices. Such was the case in COVID-19 testing, which required more than simply modifying existing scripts to address the situation (Watson \& Jeong, 2020). In many instances, nations needed to create completely new processes in order to conduct testing on the scale essential to address a pandemic. The result was the development of novel processes - such as drive-through COVID-19 testing - that required informational materials explaining each step of a new script for a new process (St.Amant, 2021). Essentially, such content provided audiences with the information needed to create new scripts based upon where a process occurred. These scripts also needed to account for who performed what tasks (and with what elements) in that location and what audiences needed to do and what to expect in such situations. 
These developments were not without problem. Rather, the creation of such content often occurred after pandemic situations led to problems where audiences diverged from existing scripts for everyday activities (Kunkle \& Ruane, 2020; Stone, 2020). Such was the case in creating new scripts for work and school (e.g., instructions on telework or online education processes) and many other common activities (e.g., online grocery shopping). Through such situations, the COVID-19 pandemic has revealed the need to address script dynamics in advance of a public health crisis whenever possible. This need is particularly acute now because many nations are transitioning back to everyday activities as COVID-19 infections decline in certain areas.

\section{Continuing Content Creation for Care}

It is one thing to apply script concepts to convey information during a public health crisis. As such threats wane, however, scripts can create different issues individuals in healthcare will need to address. Paramount among these situations is the temptation to return to doing things "as usual" prior to a public health concern (St.Amant, 2021). Essentially, individuals can be more willing to accept new processes and modify related scripts during times of crisis. Yet, when signs indicate a threat is lessening, scripts can become problematic due to how they guide behavior at subconscious level.

During times of crisis, certain markers can indicate the need to augment existing scripts in order to adapt to new threat stations (St.Amant, 2021). In such scenarios, individuals begin to view locations in new ways. During a public health crisis, for example, audiences no longer view the setting of a hospital waiting room as the location it once was. Rather, changes in that environment - alterations meant to heighten awareness of a threat - prompt individuals to identify a new location associated with a crisis. Signs noting "Please wear your mask." or "Remember to social distance." in a hospital waiting room become markers persons use to identify that setting as hospital writing room in time of crisis vs. conventional situations. This factor makes it easier for individuals to modify existing scripts associated with that setting or even create new scripts for interacting in that location.

In these situations, individuals have used crisis-related commination materials (e.g., signs) to tap the mind's script-making ability that associates particular activities with certain contexts. As a result, many of audiences used characteristics of a new environment - one associated with "in a time of crisis" - to identify a location and then produce a related script to guide their actions in that context. So, the signs that noted to "Wear your mask" or "Maintain social distance" became characteristics individuals used to identify a new location as "area in a time of crisis" (St.Amant, 2018). Individuals then created corresponding new scripts for that area in order to determine how to behave in that setting (e.g., wearing masks and socially distancing). As with 
other scripts, these associations occurred at a subconscious level, and behaviors were triggered by environmental features, like signs, that identified the location individuals were in. While these associations worked well in times of crisis, they also established different location-based scripts that can create challenges as a crisis situation changes and the markers/characteristics in an environment also change.

The issue with these dynamics is that once the elements indicating crisis situation (e.g., signs requiring individuals to wear masks) are removed from the environment, audiences begin to access the "pre-healthcare threat" scripts for a setting. Individuals then act according to the factors associated with those scripts that guided behaviors in pre-threat situations. The same could be the case for when media outlets inform audiences that restrictions for a location are no longer in place. Such information often prompts individuals to default to their prior scripts for behavior in a location by removing the need to rely on "crisis scripts." So, while wearing masks became an automatic behavior in the script for location "during a crisis," that behavior only persists as long as audiences view the related location as "during a crisis." Once that factor changes, individuals begin perceiving the location as the setting where they perform actions according to prior (i.e., pre-threat) scripts. This situation explains why many audiences around the world so easily resumed to preCOVID behaviors once authorities lifted certain restrictions or certain markers/signs were removed from public settings. These factors, however, are only one script-related challenge associated with evolving pandemic contexts.

In other cases, attempts to address public health threats during postpandemic times require the creation of new scripts for locations where audiences could perform an activity safely. In some cases, these scripts reflected completely new settings where a process occurred. The seemingly global move from physical offices to working-from-home practices caused many individuals to associate new scripts with everyday activities. These new scripts rapidly addressed an immediate public health need. The resulting situation, however, can create complications as the threat lessens and individuals begin to return to pre-pandemic settings for performing activities.

The problem is that the only script individuals have to guide their activities in such locations reflect pre-threat behaviors. Because such scripts do not contain aspects related to public safety, audiences will likely not perform important tasks - such as continuing to wear masks or socially distancing - when returning to on-site workplaces. The adoption of such prepandemic behaviors, moreover, is often instantaneous due to the subconscious nature of scripts (Ratey, 2002; Eyal, 2014). As a result, individuals could emerge from a pandemic context where they were careful about safety practices only to adopt less cautious behaviors upon returning to a familiar 
setting. Such factors could result in a new wave of infections occurring on a large scale (CBS News, 2021).

Addressing these issues is a matter of using script mapping to examine such situations in terms of scripts and associated behaviors. Content creators can then use the resulting findings to develop informational and instructional content that helps audiences either modify existing scripts or create new scripts to maintain safety in post-pandemic contexts. Such content could include instructions for new practices noting how to behave in common situations as societies re-open (e.g., scripts on how to wear masks and socially distance when returning to the workplace). This content could also involve modifying markers that identify a location in order to prompt continued or essential safety behaviors (e.g., modifying signs to note "Please continue to wear your mask to ensure the safety of those around you."). In such scenarios, the idea is that one cannot leave post-crisis situations unattended, or the scripts that caused healthcare challenges during a crisis could result in the continuation of - or even a worsening of - a situation. By using script mapping to guide content creation, different agencies within regions and across nations can address such challenges by developing healthcare content audiences can easily contextualize and readily use.

\section{Conclusion}

Societies will always need effective healthcare communication to provide audiences with essential information. The need for such content will certainly remain high as public heath crises will persist at different levels over time (Constable \& Kushner, 2021). Recent experiences with COVID-19 have revealed these dynamics in a stark way on a global level. The communication developments resulting from the COVID pandemic have emphasized the importance of understanding cognitive aspects of communication in healthcare contexts. These and other healthcare situations have revealed the importance of understanding and addressing cognitive scripts when sharing healthcare information with different audiences. By understanding the role of scripts, individuals can create textual, visual, and other content that effectively conveys information on health and medical concepts to various groups.

The ideas presented in this article provide an initial examination of such topics and offer related approaches to addressing core communication issues in healthcare contexts. Implementing these concepts will require time, effort, and patience as we map the expectations and assumptions of different audiences. Such mapping, however, can be invaluable to effective healthcare today and in the future. Essential to these practices - and to healthcare processes in the future - is realizing that human experiences shape how audiences conceptualize what healthcare is. Mapping the cognitive dynamics guiding health-related behaviors allows content creators to engage audiences 
in meaningful ways essential to both effective communication and successful healthcare practices.

\section{References:}

1. CBS News. (2021, June 23). Highly contagious Delta variant could cause next COVID-19 wave: "This virus will still find you." https://www.cbsnews.com/news/delta-variant-covid-19-contaigousnext-wave/.

2. Constable, H. \& J. Kushner. (2021). Stopping the next one: What could the next pandemic be? $B B C$.

https://www.bbc.com/future/article/20210111-what-could-the-nextpandemic-be

3. Eyal, N. (2014). Hooked: How to build habit-forming products. New York: Portfolio/Penguin Books.

4. Gavin, H. \& D. Hockey. (2010). Criminal careers and cognitive scripts: An investigation into criminal versatility. The Qualitative Report, 15(2), 389-410.

5. Hamm, R. M. (2003). Medical Decision Scripts: Combining Cognitive Scripts and Judgment Strategies to Account Fully for Medical Decision Making. In D Hardman \& L. Macchi (Eds.), Thinking: Psychological Perspectives on Reasoning, Judgement, and Decision Making (pp. 315-346). Hoboken, NJ: John Wiley \& Sons.

6. Heifferon, B. A. (2004). Writing in the health professions. New York: Pearson.

7. InterQ. (2021). Focus groups versus in-depth interviews. https://interq-research.com/focus-groups-versus-in-depth-interviews/

8. Jones. P. H. (2013). Design for care: Innovating healthcare experiences. Brooklyn, NY: Rosenfeld Media.

9. Ku, B. \& E. Lupton. (2020). Health design thinking: Creating products and services for better health. New York: Cooper Hewitt.

10. Kunkle, F., \& Ruane, M. E. (2020, March 13). Coronavirus triggers run on grocery stores, with panic-buying, hoarding and some fighting, too. The Washington Post. https://www.washingtonpost.com/dc-md$\mathrm{va} / 2020 / 03 / 13 /$ coronavirus-triggers-run-grocery-storeswith-panicbuying-hoarding-some-fighting-too/

11. Lindstrom, M. (2010). Buyology: Truth and lies about why we buy. New York: Broadway Books.

12. Pass, F., Renkl, A., \& Sweller, J. (2003). Cognitive load theory and instructional design: Recent developments. Educational Psychologist, $38(1), 1-4$.

13. Postle, B. R. (2016). How does the brain keep information "in mind"? Current Directions Psychological Science, 25(3), 151-156. 
14. Ratey, J. J. (2002). A user's guide to the brain: Perception, attention, and the four theaters of the brain. New York: Vintage.

15. St.Amant, K. (2021). Creating scripts for crisis communication: COVID-19 and beyond. Journal of Business and Technical Communication, 35(1), 126-133.

16. St.Amant, Kirk (2018) Reflexes, reactions, and usability: Examining how prototypes of place can enhance UXD practices. Communication Design Quarterly, 6(1), 45-53.

17. St.Amant, K. (2017a). Of scripts and prototypes: A two-part approach to user experience design for international contexts. Technical Communication, 64(2), 113-125.

18. St.Amant, K. (2017b). The cultural context of care in international communication design: A heuristic for addressing usability in international health and medical communication. Communication Design Quarterly, 5(2),62-70.

19. Schank, R. C., \& Abelson, R. P. (1977). Scripts, plans, goals, and understanding. Hillsdale, NJ: Erlbaum.

20. Schwab, P. N. (2016, May 19). Pros and cons of focus groups vs. interviews: an in-depth review. Into the Minds. https://www.intotheminds.com/blog/en/focus-groups-vs-interviewspros-and-cons/Social Distancing. (2020). Centers for Disease Control and Prevention. https://www.cdc.gov/coronavirus/2019ncov/prevent-getting-sick/social-distancing.html

21. Stone, W. (2020, March 24). Under Financial strain, community health centers ramp up for coronavirus response. NPR. https://www.npr.org/sections/healthshots/2020/03/24/821027067/under-financial-strain-communityhealth-centers-ramp-up-for-coronavirus-response

22. Sweller, J. (1988). Cognitive load during problem solving: Effects on learning. Cognitive Science, 12, 257-285.

23. Tomkins, Silvan S. (1987) Script theory. In J. Arnoff, A.I. Rabin, \& R.A. Zucker (Eds.), The Emergence of Personality (pp. 147-216). New York: Springer.

24. Tomkins, Silvan S. (1978) Script theory: Differential magnification of affects. Nebraska Symposium on Motivation, 26, 201-236.

25. Tse, D., Langston, R. F., Kakeyama, M., Bethus, I., Spooner, P. A., Wood, E. R., Witter, M. P., Morris, R. G. M. (2007). Schemas and memory consolidation. Science, 316(76), 76-82.

26. Watson, I. \& S. Jeong. (2020, March 3). South Korea pioneers coronavirus drive-through testing station. CNN. https://www.cnn.com/2020/03/02/asia/coronavirus-drive-throughsouth-korea-hnk-intl/index.html 
27. Yamada, R. \& Itsukushima, Y. (2013). The effects of schema on recognition memories and subjective experiences for actions and objects. Japanese Psychological Research, 55(4), 366-377.

28. Your guide to masks. (2021). Centers for Disease Control and Prevention. https://www.cdc.gov/coronavirus/2019-ncov/preventgetting-sick/about-face-coverings.html 